\title{
Study on Oil Film Characteristics of Piston-Cylinder Pair of Ultra-High Pressure Axial Piston Pump
}

\author{
Jin Zhang ${ }^{1,2,3, *}$, Baolei Liu ${ }^{1}$, Ruiqi LÜ ${ }^{1}$, Qifan Yang ${ }^{1}$ and Qimei Dai ${ }^{1}$ \\ 1 College of Mechanical Engineering, Yanshan University, Qinhuangdao 066004, China; \\ liubaoleino1@163.com (B.L.); LYURUIQI@126.com (R.L.); yqffzr@163.com (Q.Y.); \\ zhangjinno1@163.com (Q.D.) \\ 2 China Key Laboratory of Advanced Forging \& Stamping Technology and Science, Yanshan University, \\ Qinhuangdao 066004, China \\ 3 School of Mechanical Engineering, Yanshan University, Qinhuangdao 066004, China \\ * Correspondence: zhangjin@ysu.edu.cn; Tel.: +86-0335-8077-4618
}

Received: 13 December 2019; Accepted: 23 December 2019; Published: 3 January 2020

\begin{abstract}
The piston-cylinder pair is the key friction pairs in the piston pump. Its performance determines the volume efficiency of piston pump. With the increase of load pressure, the leakage at the clearance of piston-cylinder pair will also increase. In order to reduce leakage, the clearance of the piston-cylinder pair of the ultra-high pressure piston pump is smaller than that of the medium-high pressure piston pump. In order to explore whether the piston will stuck in the narrow gap, it is necessary to study the oil film characteristics of the piston-cylinder pair under the condition of ultra-high pressure, so as to provide a theoretical basis for the optimal design of the piston-cylinder pair of ultra-high pressure axial piston pump. In this paper, an ultra-high pressure axial piston pump is taken as the research object, and its structural characteristics are analyzed. The mathematical model of the oil film thickness of the piston-cylinder pair is established by using the cosine theorem in the cross section of the piston. The finite volume method is used to discretize the Reynolds equation of the oil film of the piston-cylinder pair, and the over relaxation iteration method is used to solve the discrete equations, and the mathematical model of the oil film pressure of the piston-cylinder pair is obtained. The mathematical model of oil film thickness and pressure field of piston-cylinder pair is solved by programming. The dynamic change process of oil film thickness and pressure field of the plunger pair of the ultra-high pressure axial piston pump under the load of $20 \mathrm{MPa}$ and $70 \mathrm{MPa}$ is obtained. Under the two conditions, the thinnest area of the oil film reaches $3 \mu \mathrm{m}$ and $2 \mu \mathrm{m}$ dangerous area respectively; the oil film pressure reaches $20 \mathrm{MPa}$ and $70 \mathrm{MPa}$ respectively when the swashplate rotates $10^{\circ}$ and continues to increase with the increase of swashplate rotation angle. When the rotation angle reaches $90^{\circ}$, the oil film pressure also reaches the maximum value, but there is no pressure spike phenomenon. The oil film pressure characteristics of ultra-high pressure axial piston pump under conventional and ultra-high pressure conditions were obtained by modification and experimentation.
\end{abstract}

Keywords: ultra-high pressure; axial piston pump; piston-cylinder pair; oil film characteristics; experimental study on pump

\section{Introduction}

Compared with other transmission modes, hydraulic transmission system has been widely accepted because of its advantages of large power-mass ratio, convenient control, and smooth transmission [1-3]. Ultra-high pressure is one of the development directions in hydraulic field at present. Hydraulic pump as the power component of the system, the ultra-high pressure will inevitably 
have a certain impact on its own structure [4]. During the operation of piston pump, the friction pairs bears such functions as sealing and lubrication, so the performance of the friction pairs has a key impact on the reliability and service life of the pump [5-7]. As the core component of energy conversion of piston pump, the design of piston-cylinder pair is more stringent with the ultra-high pressure of working conditions [8,9]. In order to ensure the sealing performance, the distance between piston chambers is only $3-5 \mathrm{~mm}$. Therefore, under ultra-high pressure condition, the failure of oil film of piston-cylinder pair causes many problems. For example, the wear degree between the piston-cylinder pair is aggravated, the volume efficiency is reduced, and the piston is stuck. In order to prevent the above situation of the piston-cylinder pair under ultra-high pressure condition, it is necessary to analyze the oil film characteristics of the piston-cylinder pair. In 1975, Professors Yamaguchi and Takaoka of Japan carried out theoretical and experimental analysis to explore the oil film characteristics of piston-cylinder pair. The motion of the piston is analyzed by perturbation method, and the distribution of the oil film pressure of the piston-cylinder pair is solved. A test-bed is built and the experimental results are compared with the theoretical analysis. The results are basically consistent [10]. In 1998 Tanaka et al. found that the innovation was the application of displacement sensor and force sensor. By measuring the oil film characteristics and friction characteristics of the piston-cylinder pair, the correctness of the conjecture that the piston rotates around its axis was confirmed. It was also noted that the angular velocity of the piston rotation is similar to that of the spindle [11-13]. Xiaofeng He of Huazhong University of Science and Technology established an experimental device for piston-cylinder pair of piston pump in 2001. However, because of the complexity of the motion mechanism of piston-cylinder pair, the experimental device can only be used to evaluate the wear stage of piston-cylinder pair [14]. Professor Monica I. of Purdue University, USA, has written the CASPAR program. CASPAR program is a tool for calculating the oil film characteristics of the friction pairs clearance of an anisotropic axial piston pump. The hydrodynamic, dynamic, and temperature characteristics of the oil film at the piston-cylinder pair, slipper pairs, and distributor pairs are studied. In 2009, Zhang Bin of Zhejiang University discussed the experimental method based on the oil film characteristics of the piston-cylinder pair under actual working conditions, and established the virtual prototype simulation model of the axial piston pump. The signal is sent to the data acquisition system by the sensor, and the pressure distribution of the piston oil film is tested by the piston oil film characteristic test rig. The experimental results are in good agreement with the simulation results, and the pressure field distribution of the oil film of the piston-cylinder pair can be well displayed [15]. Professor Bergada of Catalonia University of Technology in Spain has set up a test rig for dynamic oil film pressure of piston-cylinder pair. The test rig is mainly used to analyze the pressure fluctuation of the piston-cylinder pair oil film caused by the change of rotational speed, outlet pressure and inclined plate angle [16]. Professor Monica I. has built a single piston model pump experiment. The device uses a single piston pump experimental platform with swashplate rotation and fixed cylinder block to measure the oil film lubrication characteristics. Pressure distribution is measured by the pressure sensor, temperature distribution is measured by the temperature sensor, and oil film thickness is measured by the displacement sensor [17]. Xu Bing and Zhang Junhui of Zhejiang University used virtual prototyping technology to simulate the piston-cylinder pair of axial piston pump. The data transmission of sub-module was carried out by a software, and the fluid-structure coupling and rigid-flexible coupling of the piston-cylinder pair simulation model were also carried out. The validity of the model is verified by the test results, which proves that the virtual prototype simulation platform of the axial piston pump has a strong guiding role in the design of the axial piston pump [18]. Xu Bing and Zhang Junhui of Zhejiang University describe the piston state of oblique axial piston pump of electro-hydrostatic actuator accurately by solving the discrete oil film Reynolds equation and force balance equation iteratively, and obtain the leakage of piston-cylinder pair under high speed and pressure. It provides appropriate theoretical guidance for the design of EHA pump [19]. 
Nowadays, scholars from all over the world have made a detailed study of the piston-cylinder pair. However, because of the limitation of experimental conditions and the consideration of safety when the piston pump speed is too high, the pressure load setting value generally does not exceed $10 \mathrm{MPa}$. There are few studies on the oil film characteristics of piston-cylinder pair under ultra-high pressure conditions. In this paper, the oil film thickness and pressure characteristics of the piston-cylinder pair of ultra-high pressure piston pump under different working conditions are obtained through mathematical analysis and simulation analysis, and the oil film pressure characteristics of the piston-cylinder pair are obtained through experiments.

\section{Analysis of Mechanical Characteristics of Ultra-High Pressure Piston Pump}

Figure 1 shows the structure of the ultra-high pressure axial piston pump analyzed in this paper. Its rated working pressure is $70 \mathrm{MPa}$. The swashplate of the ultra-high pressure piston pump is integrated with the main shaft. It is composed of transmission shaft, pump cover, cycloid pump (refueling), pressure valve screw (valve distribution), and so on. Ultra-high pressure axial piston pump is driven by a motor to rotate the drive shaft, which causes the synchronous rotation of the swashplate and the thrust ball bearing on its surface. The return mechanism tightly compresses the piston ball head with the thrust ball bearing surface, and then drives the piston to move in the axis direction.

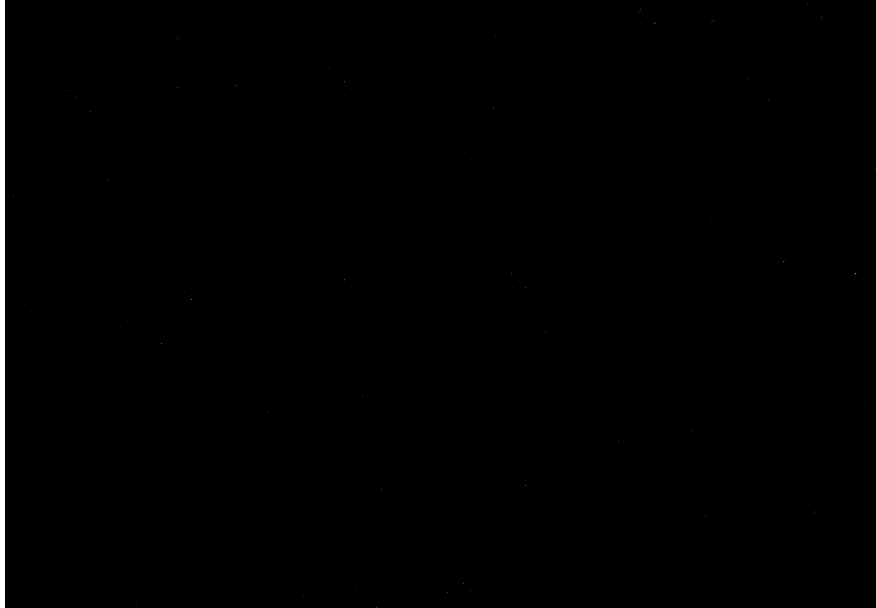

Figure 1. Structural sketch of piston pump. 1-drive shaft; 2-pump cover; 3-swash plate; 4-thrust ball bearing; 5-return mechanism; 6-piston; 7-hydraulic valve screw; 8-hydraulic cylinder; 9-marble seat; 10—lower end cover; 11—copper sleeve; 12 - cycloid pump.

The piston is subjected to hydraulic pressure of oil in the cavity, inertia force of linear motion, viscous friction force, support force of thrust ball bearing, friction force, and self-gravity. When the piston moves under their combined action, the center line of the piston and the center line of the piston cavity will not be in the same line, which will produce a certain deviation as shown in Figure 2.

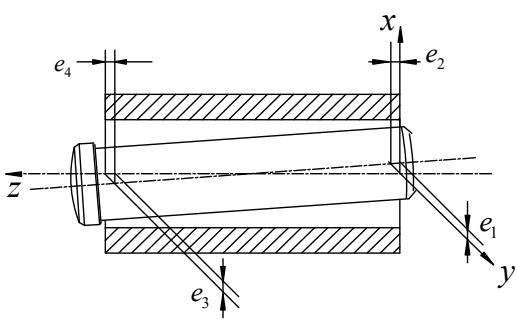

(a)

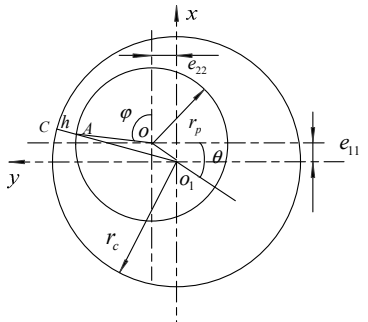

(b)

Figure 2. (a) Attitude diagram of piston in piston cavity; (b) variable schematic diagram at cross section of piston end face. 
In order to describe the inclined state of piston, the attitude inclination degree of piston in piston cavity is represented by introducing $\left(e_{1}, e_{2}, e_{3}, e_{4}\right)$ and named $\left(e_{1}, e_{2}, e_{3}, e_{4}\right)$ as offset coordinates. $\left(e_{1}, e_{2}\right)$ are near the end of the piston cavity and $\left(e_{3}, e_{4}\right)$ are near the piston ball.

The thickness of the piston-cylinder pair at any position in the axial direction of the piston satisfies:

$$
h=r_{c}-o_{1} A
$$

The unknown quantity $o_{1} A$ in the above formula can be obtained by using cosine theorem for triangle $o_{1} A$. The expression of cosine theorem is as follows:

$$
o_{1} A=\sqrt{r_{p}^{2}}+o o_{1}^{2}+2 r_{p} 0 o_{1} \sin (\varphi+\rho)
$$

In the formula, $o_{1} A$ is the distance between the center of the piston cavity section $o_{1}$ and any point $A$ on the piston section; $o o_{1}$ is the distance between the center of the piston section $o_{1}$ and the center of the piston cavity section $o_{1} ; \varphi$ is the angle between the piston center and any point on the piston and the positive direction of the $\mathrm{x}$ axis; and $o_{1}$ is the angle between the center of the piston and the center of the piston cavity and the negative direction of the $y$ axis.

Formula (2) is brought into Formula (1) to obtain:

$$
h=r_{c}-\sqrt{r_{p}^{2}+o o_{1}^{2}+2 r_{p} o o_{1} \sin (\varphi+\rho)}
$$

After simplification, we can get:

$$
h=r_{c}-r_{p}-o o_{1}+2 r_{p} \sin (\varphi+\rho)
$$

In Figure 2b, we can see that:

$$
\begin{gathered}
\tan \sigma=\frac{e_{11}}{e_{22}} \\
o o_{1}^{2}=e_{11}^{2}+e_{22}^{2}
\end{gathered}
$$

$e_{11}$ is projected in the $x$-axis direction from the center of the piston section to the center of the piston cavity, $e_{22}$ is projected in the $y$-axis direction from the center of the piston section to the center of the piston cavity.

If Formulas (5) and (6) are introduced into the simplified formula of oil film thickness, the expression $\mathrm{h}$ of oil film thickness at any point in the axial direction of piston is only a function of $e_{11}$ and $e_{22}$. Therefore, according to the values of $e_{11}$ and $e_{22}$, the oil film thickness at any position in the axial direction under the graphical attitude of piston can be obtained.

The $e_{11}$ and $e_{22}$ in the above formula are related to the migration coordinates $\left(e_{1}, e_{2}, e_{3}, e_{4}\right)$. The specific relations between them are as follows in Figure 3.

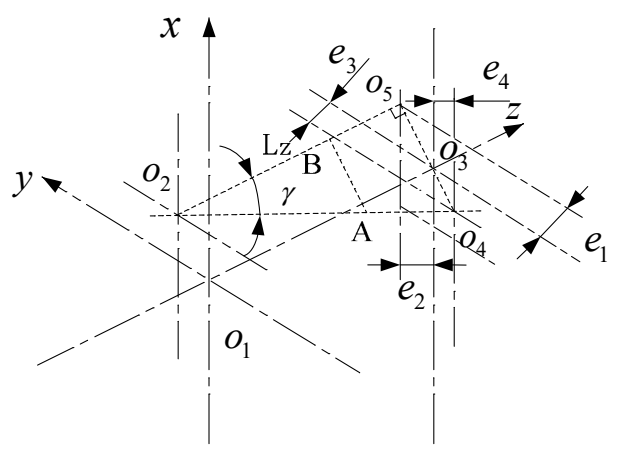

Figure 3. Diagram of relationship between displacement and displacement coordinates at piston section. 
The central deviation $\mathrm{AB}$ of any point in the axis direction of the piston:

$$
\frac{A B}{o_{4} O_{5}}=\frac{L_{Z}}{L_{C Z}}
$$

$\mathrm{AB}$ projection in horizontal and vertical directions:

$$
\begin{aligned}
& e_{11}=e_{1}-\frac{L_{Z}\left(e_{1}-e_{2}\right)}{L_{C Z}} \\
& e_{22}=e_{2}-\frac{L_{Z}\left(e_{2}-e_{4}\right)}{L_{C Z}}
\end{aligned}
$$

The mathematical model of oil film thickness of piston pairs is as follows:

$$
h=r_{c}-r_{p}-\left[e_{2}-\frac{L_{Z}\left(e_{2}-e_{4}\right)}{L_{C Z}}\right] \sin \varphi-\left[e_{1}-\frac{L_{Z}\left(e_{1}-e_{3}\right)}{L_{C Z}}\right] \cos \varphi
$$

Under the condition of ultra-high pressure, the flow of oil film in piston-cylinder pair belongs to crevice flow. Based on the theory of crevice flow and laminar flow characteristics, the following assumptions are put forward:

(1) Ignoring the proportion of mass force; (2) ignoring the inertial force of fluid; (3) ignoring the curvature of oil film, replacing rotational velocity with translation velocity; (4) ignoring the change of oil film pressure in thickness direction; (5) oil flow velocity and viscosity do not change with the change of oil film height; (6) it is assumed that the curvature radius of the interface contacted with the oil is much larger than the thickness of the oil film.

For incompressible viscous fluids, Navier-Stokes equation (N-S equation for short) characterizes the motion characteristics of fluids and is its differential equation of motion. N-S equation reflects the relationship between mass force, viscous force, and motion parameters of viscous fluid at any point in the flow process. In space rectangular coordinates, the expression of N-S equation is as follows:

$$
\left\{\begin{array}{l}
\rho f_{x}-\frac{\partial p}{\partial x}+\mu\left(\frac{\partial^{2} \mu_{x}}{\partial x^{2}}+\frac{\partial^{2} \mu_{x}}{\partial y^{2}}+\frac{\partial^{2} \mu_{x}}{\partial z^{2}}\right) \\
\rho f_{y}-\frac{\partial p}{\partial y}+\mu\left(\frac{\partial^{2} \mu_{y}}{\partial x^{2}}+\frac{\partial^{2} \mu_{y}}{\partial y^{2}}+\frac{\partial^{2} \mu_{y}}{\partial z^{2}}\right) \\
\rho f_{z}-\frac{\partial p}{\partial z}+\mu\left(\frac{\partial^{2} \mu_{z}}{\partial x^{2}}+\frac{\partial^{2} \mu_{z}}{\partial y^{2}}+\frac{\partial^{2} \mu_{z}}{\partial z^{2}}\right)
\end{array}\right.
$$

In the formula, $f_{x}$ is the mass force of a fluid with unit mass in the x-axis direction, $f_{y}$ is the mass force of a fluid with unit mass in the y-axis direction, $f_{z}$ is the mass force of a fluid with unit mass in the z-axis direction, $p$ is the fluid pressure, $\rho$ is the density of fluid, and $\mu$ is the motion viscosity of the fluid.

Under the above assumptions, the simplified N-S equation is:

$$
\left\{\begin{array}{l}
\frac{\partial p}{\partial x}=\mu \frac{\partial^{2} \mu_{x}}{\partial z^{2}} \\
\frac{\partial p}{\partial y}=\mu \frac{\partial^{2} \mu_{y}}{\partial z^{2}}
\end{array}\right.
$$

According to hypothesis (6), the annular oil film of piston pairs is expanded as follows in Figure 4.

The oil film of expanded piston-cylinder pair is analyzed in Cartesian coordinate system. Because the piston of the ultra-high pressure pump rotates in the direction of the non-winding transmission shaft, and because the motion between the swashplate and the cylinder block is reciprocal. Therefore, in order to express the velocity boundary condition of the oil film of the piston-cylinder pair, the actual situation of cylinder block fixing and swashplate rotating are transformed into that of cylinder block rotating and swashplate fixing. 


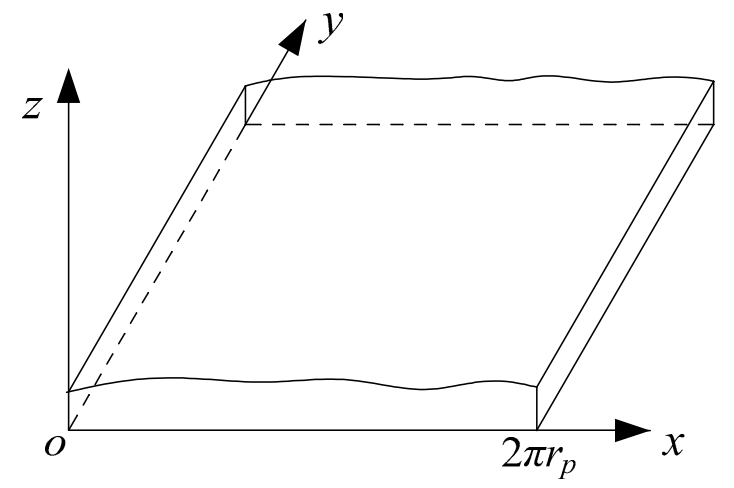

Figure 4. Piston pairs oil film unfolding diagram.

Mathematical model expression of oil film pressure of piston pairs:

$$
\frac{\partial}{\partial x}\left(\frac{\partial P}{\partial x} \frac{h^{3}}{\mu}\right)+\frac{\partial}{\partial y}\left(\frac{\partial P}{\partial y} \frac{h^{3}}{\mu}\right)=6 r_{p} \omega_{p} \frac{\partial h}{\partial x}+6 v_{p} \frac{\partial h}{\partial y}+12 \frac{\partial h}{\partial t}
$$

In the formula: $h$ is the thickness of the oil film of the piston-cylinder pair; $\mu$ is the viscosity of the oil film of the piston-cylinder pair; $P$ is the pressure of the oil film of the piston-cylinder pair.

In view of the very small film thickness of the ultra-high pressure piston pump, in order to simplify the solution process, the expanded oil film is equivalent to a plane form. In this paper, the Reynolds equation of oil film is solved by the finite volume method. The expanded oil film is meshed as follows (Figure 5a). By integrating the Reynolds equation of oil film in each control volume, a set of discrete equations about pressure can be solved. Therefore, by solving the discrete equations, the pressure distribution at each point on the oil film of the piston-cylinder pair under ultra-high pressure can be obtained. Establish a two-dimensional control volume structure as shown in Figure 5b.

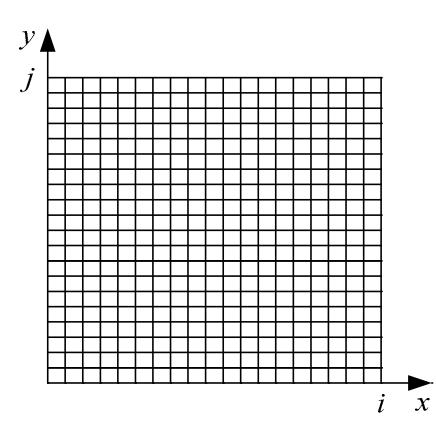

(a)

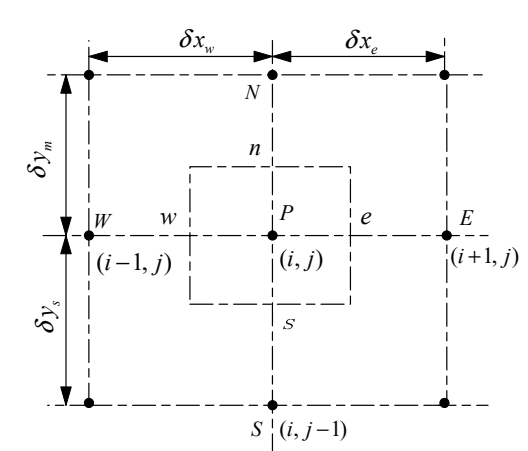

(b)

Figure 5. (a) Schematic diagram of oil film plane mesh generation; (b) control volume structure diagram.

According to the idea of finite volume method, the Reynolds equation of oil film is integrated into the control volume n-w-s-e in Figure 5b, which is expressed as follows:

$$
\int_{V}\left(\frac{\partial}{\partial x}\left(\frac{\partial P}{\partial x} \frac{h^{3}}{\mu}\right)+\frac{\partial}{\partial y}\left(\frac{\partial P}{\partial y} \frac{h^{3}}{\mu}\right)\right) d V=\int_{V}\left(6 r_{p} \omega_{p} \frac{\partial h}{\partial x}+6 v_{p} \frac{\partial h}{\partial y}+12 \frac{\partial h}{\partial t}\right) d V
$$

The volume integral of formula is transformed into area integral by using the Gauss theorem. The expression of the Gauss theorem is as follows:

$$
\int_{s}^{n} \int_{w}^{e}\left(\frac{\partial p}{\partial x}+\frac{h^{3}}{\mu}\right) d x d y+\int_{s}^{n} \int_{w}^{e}\left(\frac{\partial p}{\partial y} \frac{h^{3}}{\mu}\right) d x d y-\oiiint\left(6 r_{p} w_{p} \frac{\partial h}{\partial x}+6 v_{p} \frac{\partial h}{\partial y}+12 \frac{\partial h}{\partial t}\right) d x d y=0
$$


In the control volume $n-w-s-e$, the horizontal e and $w$ points are:

$$
\left(\frac{\partial p}{\partial x} \frac{h^{3}}{\mu}\right)_{e}=\frac{h_{e}^{3}}{\mu} \frac{P_{N}-P_{P}}{\delta x_{P E}},\left(\frac{\partial p}{\partial x} \frac{h^{3}}{\mu}\right)_{w}=\frac{h_{w}^{3}}{\mu} \frac{P_{P}-P_{W}}{\delta x_{W P}}
$$

In the control volume n-w-s-e, the vertical $n$ and s points are:

$$
\left(\frac{\partial p}{\partial x} \frac{h^{3}}{\mu}\right)_{n}=\frac{h_{n}^{3}}{\mu} \frac{P_{N}-P_{P}}{\delta x_{N P}},\left(\frac{\partial p}{\partial x} \frac{h^{3}}{\mu}\right)_{S}=\frac{h_{s}^{3}}{\mu} \frac{P_{P}-P_{N}}{\delta x_{P S}}
$$

Bring the formula into the formula and simplify it into:

$$
\begin{gathered}
a_{P} P_{P}=a_{N} P_{N}+a_{S} P_{S}+a_{E} P_{E}+a_{W} P_{W}+S \\
a_{P}=a_{N}+a_{S}+a_{E}+a_{W} \\
a_{N}=\frac{h_{n}^{3}}{\mu} \frac{\delta x}{\delta y}, a_{S}=\frac{h_{s}^{3}}{\mu} \frac{\delta x}{\delta y}, a_{E}=\frac{h_{e}^{3}}{\mu} \frac{\delta x}{\delta y}, a_{W}=\frac{h_{w}^{3}}{\mu} \frac{\delta x}{\delta y} \\
S=-6\left(\omega r_{p}\left(h_{e}-h_{w}\right) \delta y-v_{p}\left(h_{n}-h_{S}\right) \delta x\right)-12 \int_{w}^{e} \int_{s}^{n} h^{\prime} d x d y
\end{gathered}
$$

In the formula, $h_{n}$ is the oil film thickness at $n$ point in n-w-s-e, $h_{s}$ is the oil film thickness at $s$ point in n-w-s-e, $h_{e}$ is the oil film thickness at $e$ point in n-w-s-e, and $h_{w}$ is the oil film thickness at $n$ point in $\mathrm{n}-\mathrm{w}-\mathrm{s}-\mathrm{e}, \delta \mathrm{x}$ is the $\mathrm{x}$-direction length of each micro-grid in the oil film division area of the piston-cylinder pair; $\delta y$ is the $y$-direction length of each micro-grid in the oil film division area of the piston-cylinder pair; $h^{\prime}$ is the change rate of the oil film thickness of the piston-cylinder pair with time; and $\mu$ is the motion viscosity of the fluid.

In this paper, the successive over-relaxation iteration method (SOR iteration method) is selected to solve the discrete equation. Over-relaxation method is a modification of the Gauss-Seidel algorithm (GS algorithm). In the iteration process, the results of each iteration and the changes of each iteration are weighted and then brought into the next calculation. Therefore, successive over-relaxation iteration method greatly optimizes the convergence rate. The oil film discrete equation of the piston pairs is substituted into the iteration equation to obtain:

$$
P_{p}^{k+1}=\omega\left(\frac{a_{N} P_{N}^{k}+a_{S} P_{S}^{k}+a_{W} P_{W}^{k}+a_{E} P_{E}^{k}+S}{a_{P}}-P_{P}^{k}\right)+P_{P}^{k}
$$

In this paper, the convergence criteria for discrete computation of equations are as follows:

$$
\frac{\sum_{i=2}^{m-1} \sum_{j=1}^{n}\left|P_{i, j}^{k}-P_{i, j}^{k-1}\right|}{\sum_{i=2}^{m-1} \sum_{j=1}^{n}\left|P_{i, j}^{k}\right|} \leq \text { error }
$$

In order to ensure better convergence and calculation accuracy, the convergence accuracy error $=10^{-3}$ and the value range of relaxation factor $\omega$ are chosen between 1.6 and 1.8.

\section{Simulation and Analysis of Oil Film Characteristics of Ultra-High Pressure Axial Piston Pump}

In this paper, the oil film characteristics of ultra-high pressure axial piston pump are simulated and analyzed by using the simulation software of MATLAB. In order to compare the oil film characteristics of piston-cylinder pair under ultra-high pressure with those of ordinary medium and high pressure piston pumps, the oil film characteristics of piston-cylinder pair were simulated under $20 \mathrm{MPa}$ and $70 \mathrm{MPa}$ respectively. Parameter settings are shown in Table 1. 
Table 1. Simulation parameter setting.

\begin{tabular}{cc}
\hline Parameter & Numerical Value \\
\hline Piston cavity radius mm & 4.005 \\
Piston radius mm & 4 \\
Number of pistons & 13 \\
Bushing length mm & 41 \\
Piston distribution circle radius mm & 100 \\
Swash plate inclination angle ${ }^{\circ}$ & 7 \\
Piston quality kg & $195.8 \times 10^{-3}$ \\
Longest contact length of piston pairs mm & 34.3 \\
Distance from piston center to end mm & 48 \\
Speed of piston pump r/min & 1500 \\
Pressure of oil chamber MPa & 70 \\
Suction chamber pressure MPa & 2.5 \\
Pressure inside the shell MPa & 0.2 \\
Oil viscosity Pa·s & 0.046 \\
Axial meshing number & 40 \\
Number of circumferential meshes & 40 \\
Pressure of oil chamber MPa & 70 \\
Suction chamber pressure Mpa & 2.5 \\
Pressure in shell MPa & 0.2 \\
Oil viscosity Pa·s & 0.046 \\
Axial grid fraction & 40 \\
Circumferential grid fraction & 40 \\
\hline
\end{tabular}

Under constant pressure (20 MPa), the oil film thickness expression of the piston-cylinder pair obtained above is brought into the simulation software of MATLAB, and the results are shown in Figure 6 (the point of coordinate $(0,0)$ in the figure below corresponds to point A in Figure $2 b$ ).

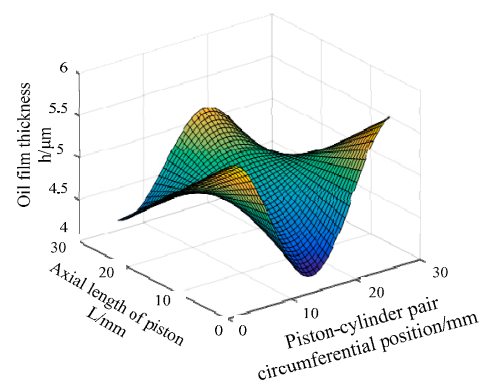

(a)

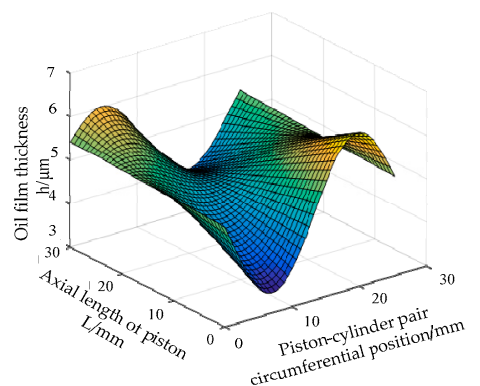

(d)

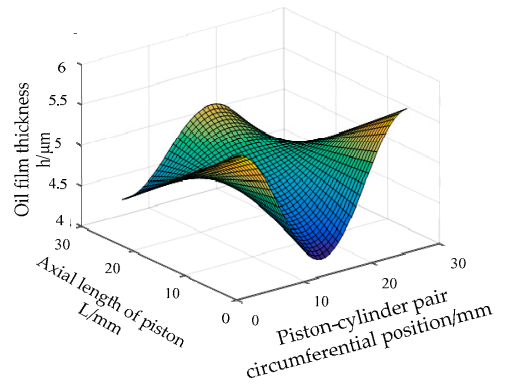

(b)

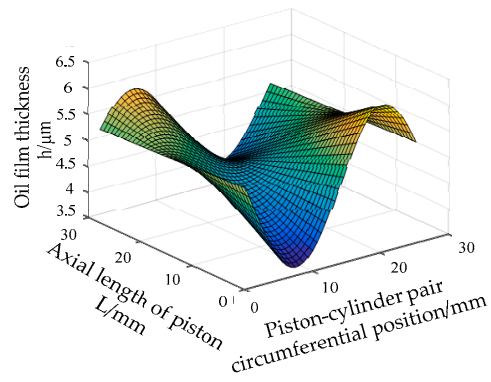

(e)

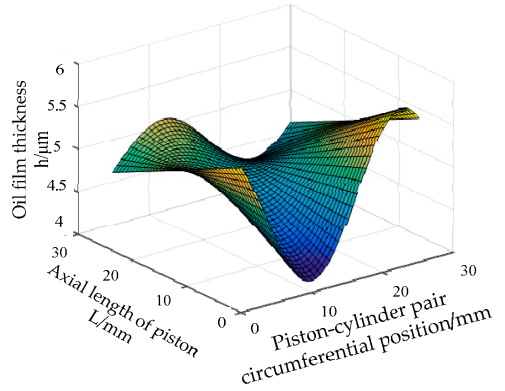

(c)

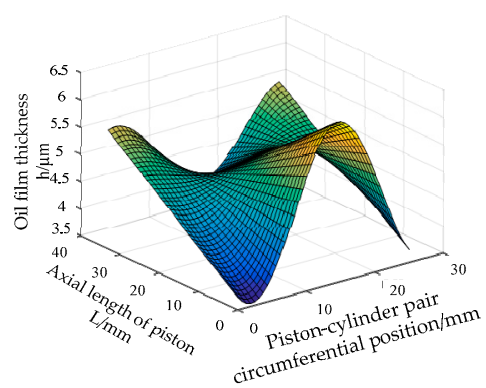

(f)

Figure 6. The figure shows the result of oil film thickness at different angle of inclined plate rotation: (a) Oil film thickness distribution with swashplate rotation of $1^{\circ} ;(\mathbf{b})$ oil film thickness distribution with swashplate rotation of $10^{\circ}$; (c) oil film thickness distribution with swashplate rotation of $45^{\circ}$; (d) oil film thickness distribution with swashplate rotation of $90^{\circ}$; (e) oil film thickness distribution with swashplate rotation of $110^{\circ}$; (f) oil film thickness distribution with swashplate rotation of $210^{\circ}$. 
When the swashplate rotates $1^{\circ}$, the plunger is still near the starting point, the inclination of the plunger is very small, and the oil film thickness is about $5 \mu \mathrm{m}$. When the swashplate rotates $10^{\circ}$, the plunger cavity enters the oil pressure area, and the pressure in the plunger cavity increases, resulting in the increase of the eccentric load on the plunger and the increase of the inclination of the plunger. When the swashplate rotates $45^{\circ}$, the eccentric load on the plunger continues to increase, resulting in the increase of the inclination of the plunger. When the swashplate rotates $90^{\circ}$, the movement speed of the plunger reaches the maximum. At this time, the eccentric load of the plunger is the maximum, the inclination degree of the plunger reaches the maximum, the oil film thickness of the cylinder-piston pair reaches the minimum, and the oil film thickness is $3-3.5 \mu \mathrm{m}$. When the swashplate rotates $110^{\circ}$, the eccentric load on the plunger is relieved and the inclination of the plunger is reduced. When the swashplate rotates $210^{\circ}$, the plunger enters into the low-pressure oil absorption area, and the degree of eccentric load of the plunger is very small, and the oil film distribution of the cylinder-piston pair is relatively average.

Under constant pressure (20 MPa), the expression of the oil film pressure gauge of the piston-cylinder pair obtained above is brought into the simulation software of MATLAB, and the results are shown in Figure 7.

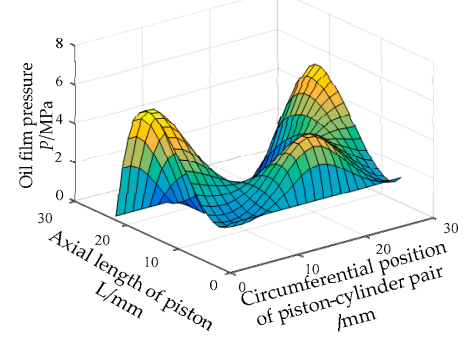

(a)

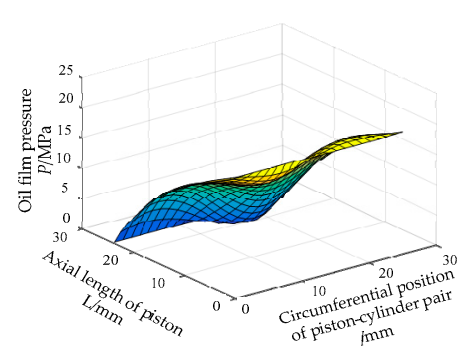

(d)

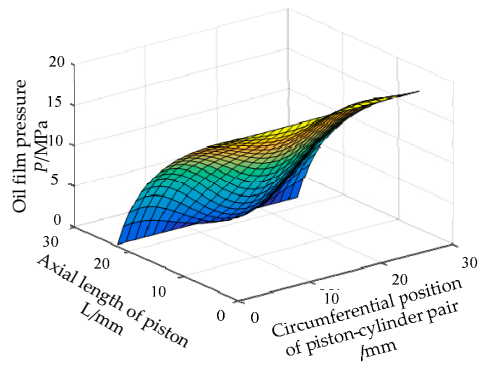

(b)

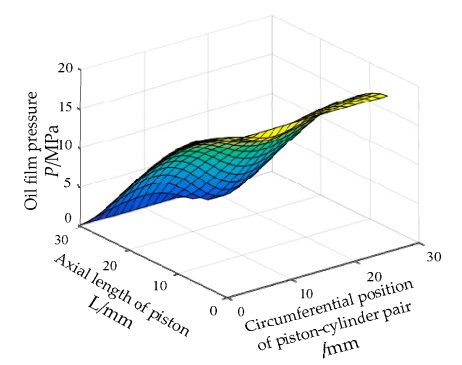

(e)

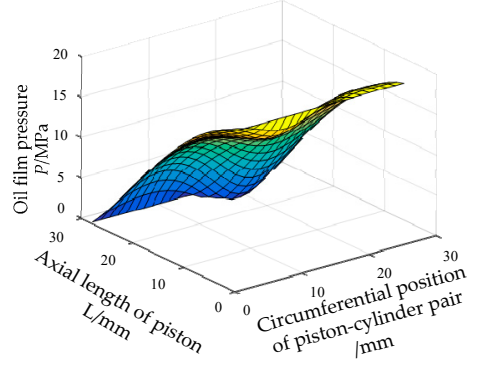

(c)

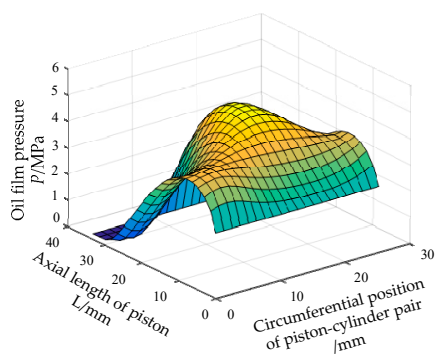

(f)

Figure 7. The figure shows the distribution of oil film pressure at different inclined plate rotation angles under constant pressure conditions: (a) Oil film pressure distribution with swashplate rotation of $1^{\circ}$; (b) oil film pressure distribution with swashplate rotation of $10^{\circ}$; (c) oil film pressure distribution with swashplate rotation of $45^{\circ}$; (d) oil film pressure distribution with swashplate rotation of $90^{\circ}$; (e) oil film pressure distribution with swashplate rotation of $110^{\circ}$; (f) oil film pressure distribution with swashplate rotation of $210^{\circ}$.

When the swashplate rotates $1^{\circ}$, because the plunger is still near the starting point, the pressure in the plunger cavity is not established and the pressure is small. When the swashplate rotates for $10^{\circ}$, the plunger cavity enters the oil pressure area, and the inclination degree of the plunger increases, so the oil film pressure reaches $20 \mathrm{MPa}$. When the swashplate rotates $45^{\circ}$, with the increase of the pressure in the plunger cavity, the inclination degree of the plunger continues to increase. Therefore, in order to balance the increased eccentric load of the plunger, the oil film pressure continues to increase. When the swashplate rotates to $90^{\circ}$, the movement speed of the plunger is the maximum, the eccentric load degree of the plunger reaches the maximum, and the oil film appears the thinnest area in the 
whole movement cycle. Therefore, in order to balance the eccentric load, the oil film pressure field of the cylinder-piston pair reaches the maximum, and the oil film pressure reaches $25 \mathrm{MPa}$. When the swashplate rotates to $110^{\circ}$, the eccentric load on the plunger decreases and the pressure field of the oil film decreases. When the swashplate rotates $210^{\circ}$, the plunger cavity has left the oil pressure area and stepped into the low-pressure oil absorption area. The plunger tends to be in a stable state. Therefore, the oil film pressure distribution is small. The oil film characteristics of the ultrahigh pressure plunger pump studied in this paper are basically the same as those in reference [15]. When pressing oil, when the inclination of plunger increases, the oil film pressure increases, and when the inclination of plunger decreases, the oil film pressure also decreases. In the process of oil absorption, the pressure distribution of the plunger is also small because of its small inclination. However, the oil film gap of the cylinder-piston pair in this paper is only $5 \mu \mathrm{m}$, which is quite different from the oil film gap $(17 \mu \mathrm{m})$ in reference [15]. Therefore, although the inclination degree of the plunger will increase, the space $(5 \mu \mathrm{m})$ for the inclination of the plunger is not as large as that in reference [15] (17 $\mu \mathrm{m})$. Therefore, the oil film of the cylinder-piston pair in this paper is squeezed, but the oil film is squeezed membrane pressure does not have a sharp edge. The pressure spike shown in Figure 8 does not occur.

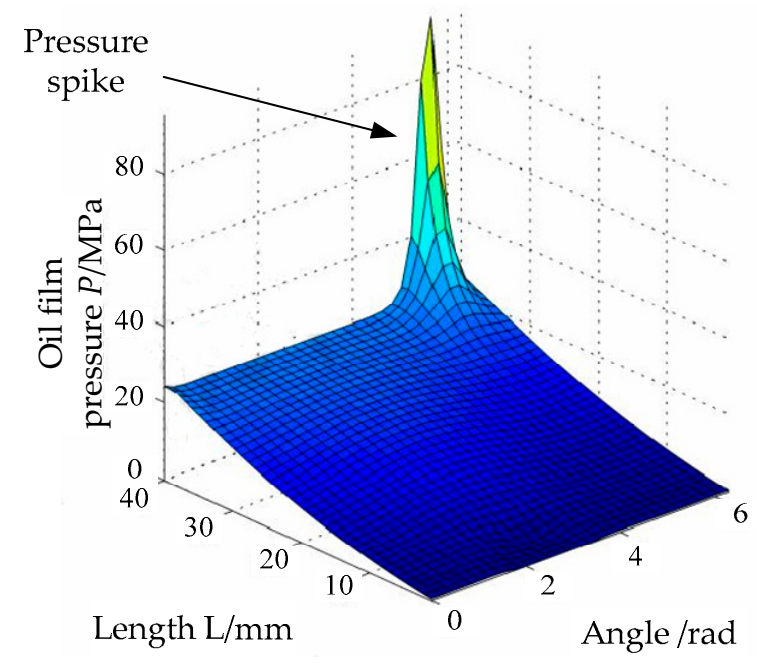

Figure 8. The figure shows pressure spike of plunger auxiliary oil film.

Under the condition of ultra-high pressure $(70 \mathrm{MPa})$, the oil film thickness expression of the piston-cylinder pair obtained above is brought into the simulation software of MATLAB, and the results are shown in Figure 9.

Compared with the normal pressure condition, the change trend and distribution of the oil film thickness under the ultra-high pressure condition are the same. Before the swashplate rotates $45^{\circ}$, the oil film thickness is almost the same. But after the swashplate rotates $45^{\circ}$, the oil film thickness under the ultra-high pressure condition is obviously smaller than that under the normal pressure condition. The minimum thickness of oil film is about $2 \mu \mathrm{m}$ under ultra-high pressure condition. At this time, the bearing limit area appears in the auxiliary oil film of the plunger. The inclination degree of the plunger is greater than that under normal pressure condition, and the failure probability of the auxiliary oil film of the plunger is greater. When the swashplate rotates more than $90^{\circ}$, the oil film thickness under the two conditions is basically the same.

Under the condition of ultra-high pressure $(70 \mathrm{MPa})$, the expression of the oil film pressure gauge of the piston-cylinder pair obtained above is brought into the simulation software of MATLAB, and the results are shown in Figure 10. 


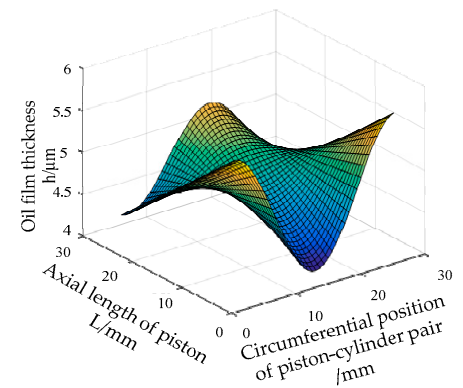

(a)

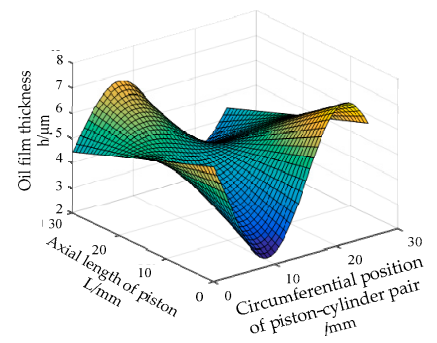

(d)

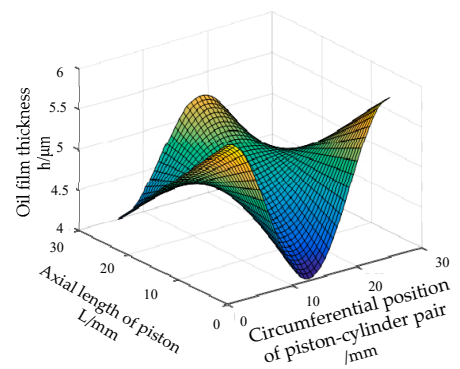

(b)

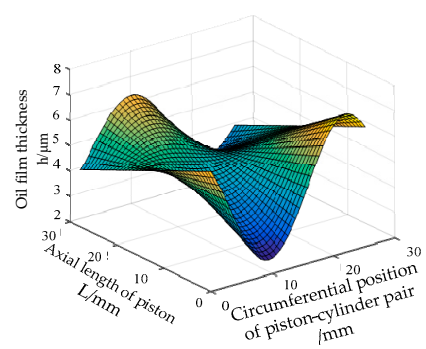

(e)

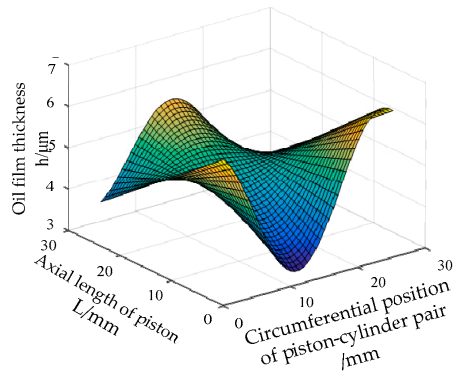

(c)

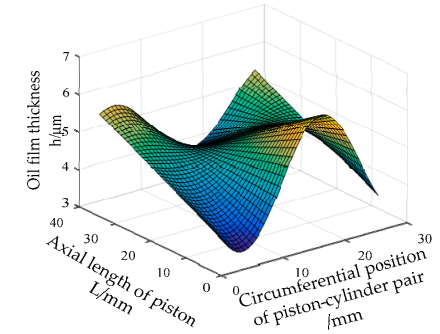

(f)

Figure 9. The figure shows the distribution of oil film thickness under different inclined plate rotation angles under ultra-high pressure conditions: (a) Oil film thickness distribution with inclined disk rotation of $1^{\circ} ;(\mathbf{b})$ oil film thickness distribution with inclined disk rotation of $10^{\circ}$; (c) oil film thickness distribution with inclined disk rotation of $45^{\circ}$; (d) oil film thickness distribution with inclined disk rotation of $90^{\circ}$; (e) oil film thickness distribution with inclined disk rotation of $110^{\circ}$; (f) oil film thickness distribution with inclined disk rotation of $210^{\circ}$.

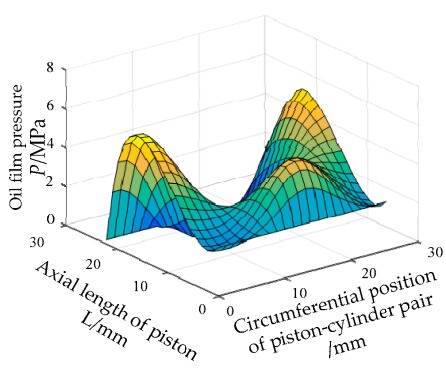

(a)

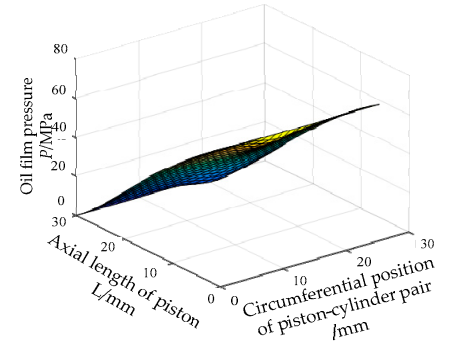

(d)

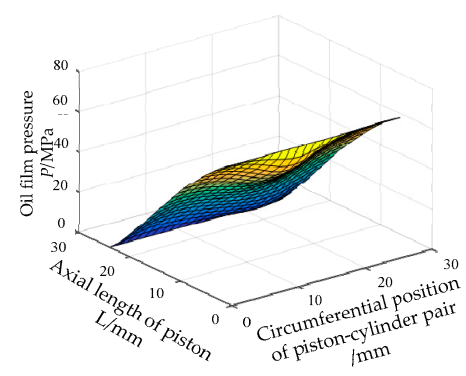

(b)

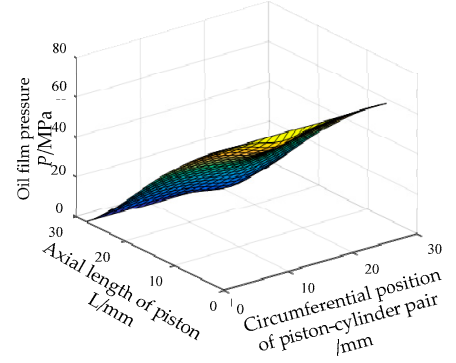

(e)

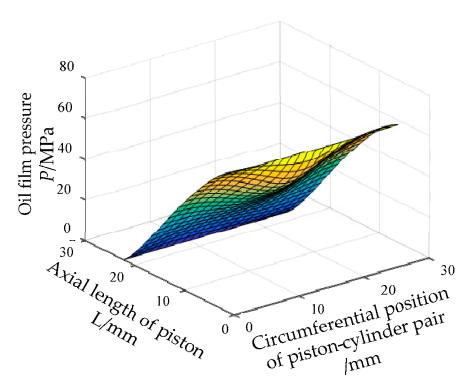

(c)

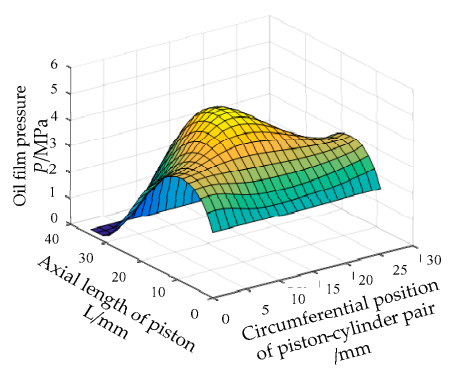

(f)

Figure 10. The figure shows the distribution of oil film pressure under different inclined plate rotation angles under ultra-high pressure conditions: (a) Oil film pressure distribution with swashplate rotation of $1^{\circ} ;(\mathbf{b})$ oil film pressure distribution with swashplate rotation of $10^{\circ}$; (c) oil film pressure distribution with swashplate rotation of $45^{\circ}$; (d) oil film pressure distribution with swashplate rotation of $90^{\circ}$; (e) oil film pressure distribution with swashplate rotation of $110^{\circ}$; (f) oil film pressure distribution with swashplate rotation of $210^{\circ}$. 
The change trend of oil film pressure under the condition of ultra-high pressure is the same as that under the condition of normal pressure, but there is a big difference in the value. The oil film pressure under the condition of ultra-high pressure is far greater than that under the condition of normal pressure, In this paper, the oil film gap of the cylinder-piston pair of the ultra-high pressure pump is only $5 \mu \mathrm{m}$, so whether the oil film thickness is squeezed to $3 \mu \mathrm{m}$ under the normal pressure condition or the oil film gap is squeezed to $2 \mu \mathrm{m}$ under the ultra-high pressure condition, the piston tilt space of the two is not large, so there is no pressure spike phenomenon that the normal pressure piston pump will appear when the swashplate rotates $90^{\circ}$.

The oil film thickness and pressure distribution trend of the piston-cylinder pair under $70 \mathrm{MPa}$ condition are basically the same as that under $20 \mathrm{MPa}$ condition. All of them are as follows: when oil is pressed, the axial force of piston increases with the increase of the angle of inclined plate rotation, which leads to the increase of eccentric load on the piston, the increase of piston tilt, and the increase of oil film pressure; when the swashplate rotates at $90^{\circ}$, the axial velocity and force of the piston are the largest, and the eccentric load of the piston is the most serious. The dangerous area of the oil film thickness is less than $3 \mu \mathrm{m}$, and the oil film pressure reaches the maximum at this time; when the swashplate rotates at $125^{\circ}$, the axial force acting on the piston is relieved, the inclination degree of the piston is relatively reduced, and the oil film pressure is also reduced. When the piston chamber is sucking oil, the piston tends to be stable and the oil film pressure distribution is small.

\section{Experimental Study on Film Pressure of Piston Pairs in Ultra-High Pressure Axial Piston Pump}

Because the oil film gap of piston-cylinder pair is very small, the existence of processing errors and the difficulty of installing pressure sensors lead to the measurement of oil film thickness is difficult and the reliability is low, so this paper only tests the oil hydraulic pressure. In the experiment, we choose the way of reforming the solid pump to analyze the oil film of the cylinder-piston pair, and directly measure the pressure of the oil film pair on the solid pump. When selecting the experimental pressure measurement points, the more intensive the experimental measurement points are selected, the more representative the comparison is with the simulation results. Because of the structure of the pump itself, limited by the installation position, the volume of the sensor itself, and the existence of the oil outlet check valve, only one pressure sensor can be installed on the cylinder body for each plunger. In this paper, three pressure measuring points are actually selected on the cylinder block to measure the axial pressure of three different plungers in the same position. Finally, the pressure measurement results of the three measuring points on the cylinder are compared with the simulation results. Figure 11 is the schematic diagram of the measurement of the oil film pressure of the cylinder-piston pair.
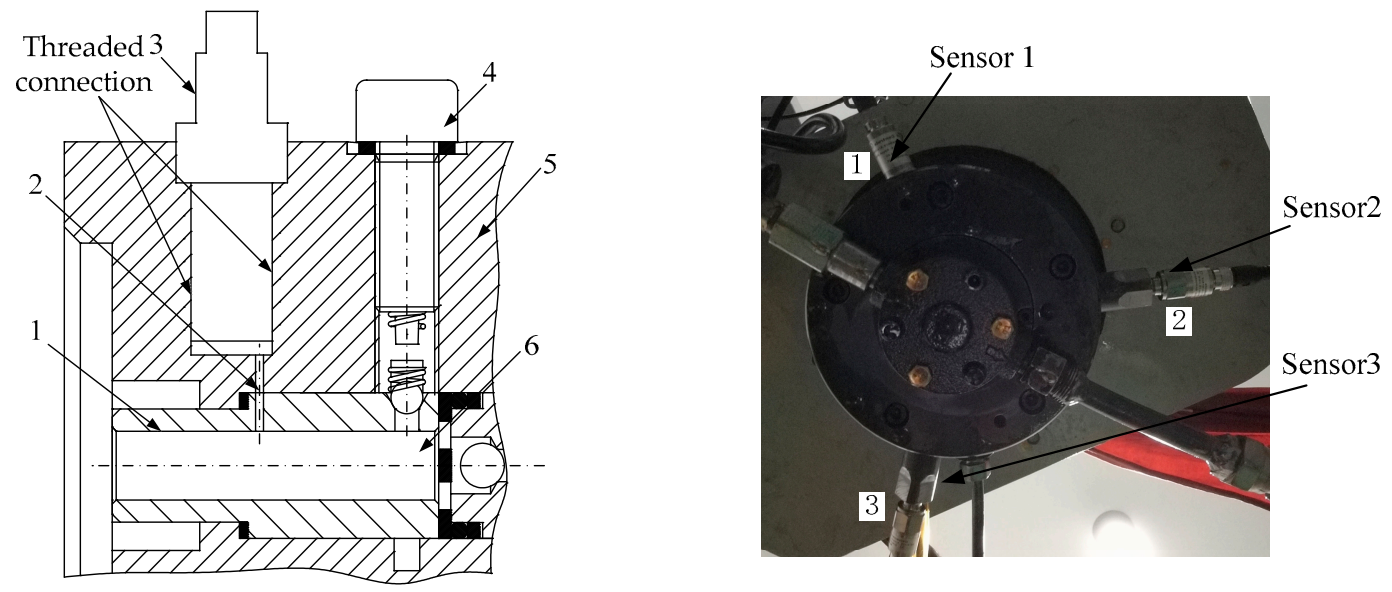

Figure 11. Schematic diagram of plunger auxiliary oil film pressure measurement. 1 -plunger sleeve; 2-oil drain hole; 3 - pressure sensor; 4-pressure valve screw; 5 -cylinder block; 6-plunger cavity. 
In this section, an experimental platform is designed for the pump, which can provide different load pressures. The experimental platform can complete the measurement of the oil film pressure of the piston-cylinder pair under different load pressures. The principle of the experimental platform is shown in the Figure 12.

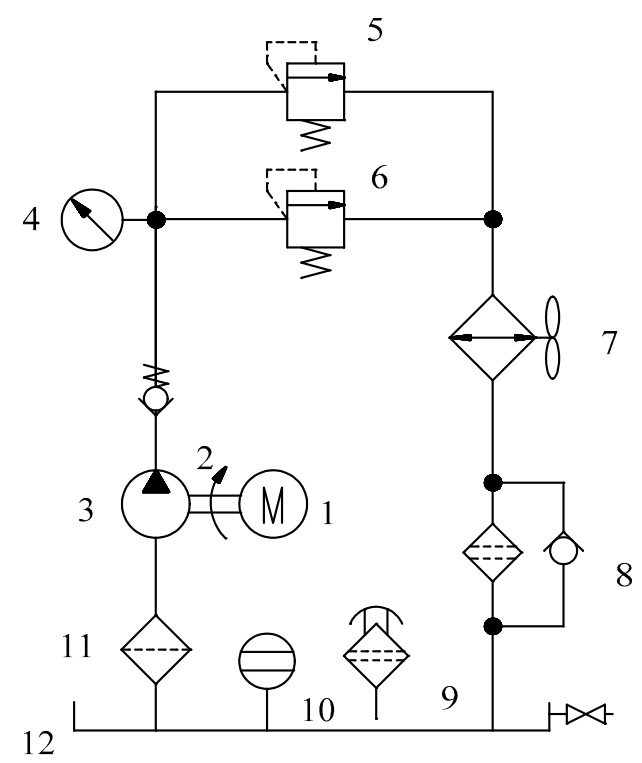

Figure 12. The figure is the schematic diagram of the test bed. 1 -motor; 2 -coupling; 3 -pump; 4-pressure gauge; 5 -high pressure relief valve; 6-relief valve (relief valve); 7—air cooler; 8-oil return filter; 9-air filter; 10-level gauge; 11—filter; 12-fuel tank.

The assembled test device is shown in Figure 13.

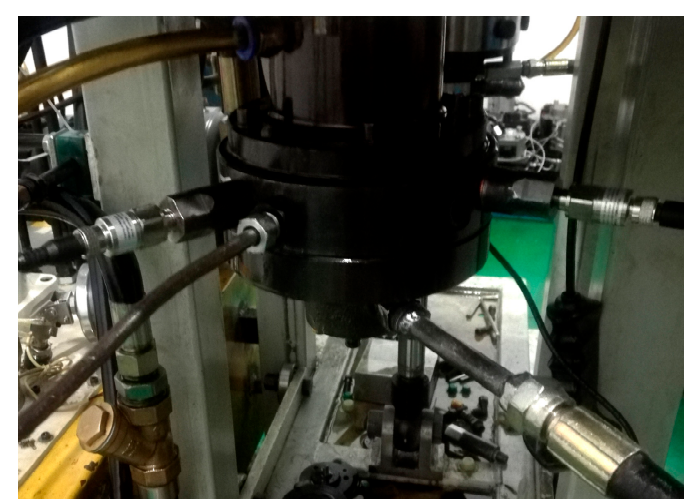

(a)

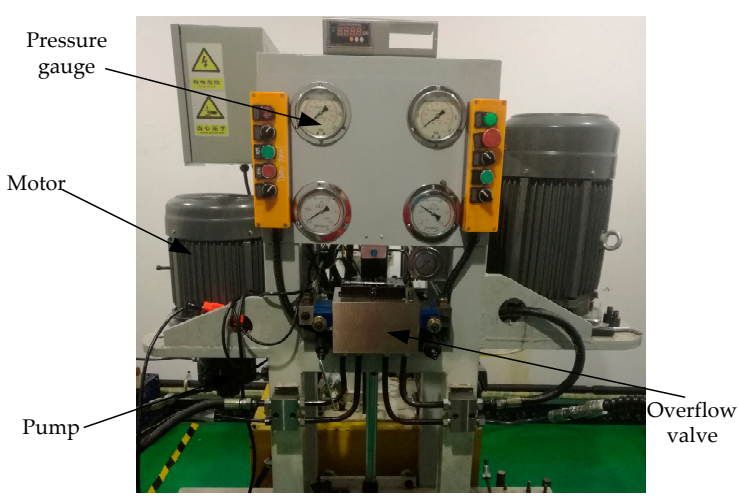

(b)

Figure 13. The figure above shows the experimental object: (a) Physical drawing of pump; (b) physical drawing of pump test bed.

This paper uses NI industrial computer and multi-functional data acquisition system, pressure sensor, measurement and acquisition software LabVIEW, and data processing and analysis in data acquisition software LabVIEW.

The experimental results are shown in Figure 14. 


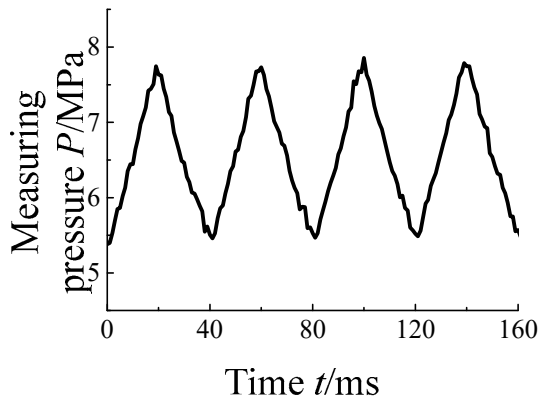

(a)

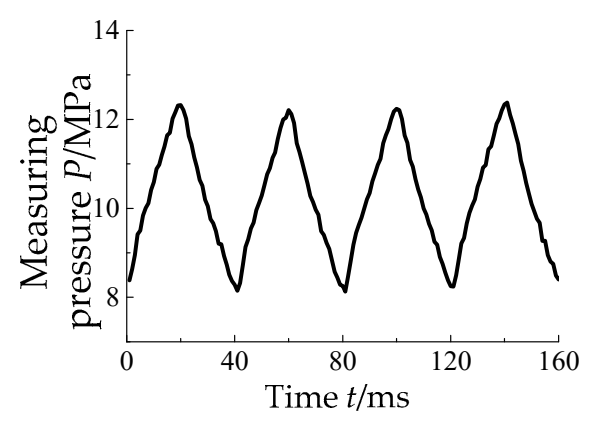

(b)

Figure 14. This figure is the experimental result: (a) Change of oil film pressure under $20 \mathrm{MPa}$ load; (b) change of oil film pressure under $70 \mathrm{MPa}$ load.

In order to better compare the test results with the simulation, this paper also simulates the oil film pressure of the plunger pair under the condition of the rated speed of $1500 \mathrm{r} / \mathrm{min}$ and the load pressure of $20 \mathrm{MPa}$ and $70 \mathrm{MPa}$. According to the position of the measurement point in the test, the pressure at the simulation point is connected into a curve, and the simulation pressure curve is shown in the Figure 15.

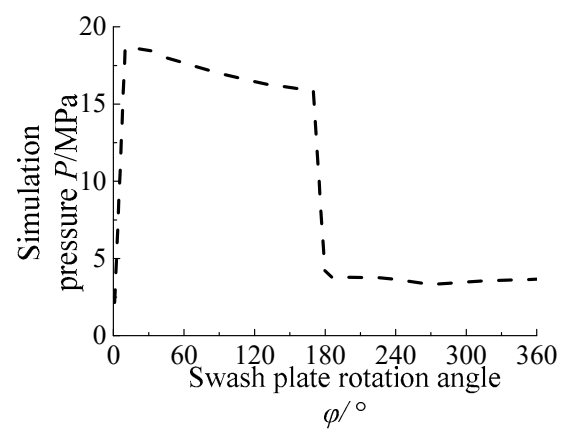

(a)

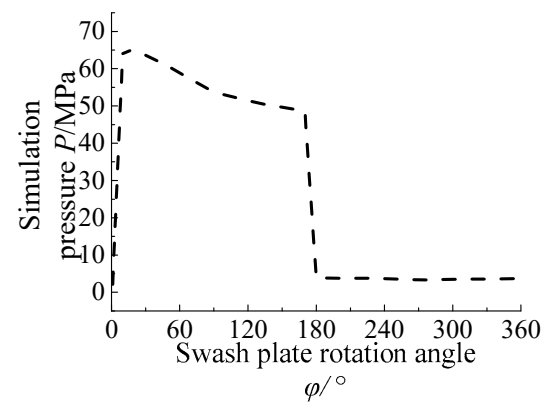

(b)

Figure 15. This figure is the simulation result: (a) Simulation curve of oil film pressure with load pressure of $20 \mathrm{MPa}$; (b) simulation curve of oil film pressure with load pressure of $70 \mathrm{MPa}$.

According to test Figure 14 and simulation Figure 15, the following conclusions can be obtained: (1) The change trend of the test pressure curve is basically the same as that of the simulation pressure curve, showing the trend of first increasing and then decreasing; (2) the test pressure curve shows a triangular trend, and the simulation pressure curve shows a trapezoid like trend, that is to say, when the test pressure curve is pressurized, the pressure rises slowly, and at the same time, it is unable to hold the high pressure; (3) with the increase of the load pressure, the pressure values measured in the test are less than the simulation pressure. The larger the load pressure is, the greater the difference between the pressure values obtained in the test and the simulation results is.

The main reason for the above (2) and (3) phenomena is that the oil drawing hole is processed too much. In the measurement process, when the oil reaches the bottom of the threaded hole where the pressure sensor is installed through the oil drain hole, the excessive diameter of the oil drain hole will cause the oil to leak along the wall of the threaded hole, causing a large pressure loss and slow pressure growth. In the process of oil pressure, the larger the load pressure is, the larger the oil leakage through the threaded hole wall of the pressure sensor is, and the larger the pressure measurement error is. Therefore, in the test process, with the increase of load pressure, the measured pressure values are smaller. 


\section{Conclusions}

(1) The attitude of piston in piston chamber under ultra-high pressure is described, and the mathematical model of oil film thickness of piston-cylinder pair is established. The fluid continuity equation and N-S equation are combined, and Reynolds equation is discretized by finite volume method. The mathematical model of oil film pressure of piston-cylinder pair under ultra-high pressure (70 MPa) is obtained.

(2) The dynamic characteristics of oil film of piston-cylinder pair are simulated and analyzed under $20 \mathrm{MPa}$ and $70 \mathrm{MPa}$ respectively. The distribution of oil film pressure and thickness at different rotating angles is obtained. The oil film pressure reaches the maximum when the inclined plate rotates to $90^{\circ}$ and the oil film thickness reaches the minimum at this time. Under $70 \mathrm{MPa}$, the oil film thickness reaches $2 \mathrm{~mm}$ when the oil film thickness is the minimum, and at this time the oil film thickness reaches $2 \mathrm{~mm}$. Oil film failure occurs easily when the pressure is higher than $70 \mathrm{MPa}$.

(3) The dynamic value of the oil film pressure of the piston-cylinder pair was collected and compared with the simulation results. It was found that the change trend of the oil film test pressure curve was basically the same as that of the simulation pressure curve, which increased first and then decreased. However, there is a gap between the measured pressure and the simulation because of the processing error of the oil intake hole in the test process.

Author Contributions: Conceptualization, J.Z.; methodology, J.Z., B.L.; experiments, Q.D., R.L.; data analysis, B.L., Q.Y.; supervision, Q.Y., R.L.; writing—review and editing, J.Z., B.L., R.L., and Q.Y. All authors have read and agreed to the published version of the manuscript.

Funding: This research was supported by the National Natural Science Foundation of China project (51805467).

Conflicts of Interest: The authors declare no conflicts of interest.

\section{References}

1. Wang, Z.J. Development Status and Trend of Hydraulic Technology. J. Appl. Technol. 2016, 20, 85-89.

2. Li, G.B.; Han, L.W.; Han, B. Status and developing trend of hydraulic technology. J. Jiangsu Sci. Technol. Inf. 2016, 24,74 .

3. Manring, D.; Mehta, S.; Nelson, B.E.; Graf Kevin, J.; Kuehn Jeff, L. Increasing the Power Density for Axial-Piston Swash-Plate. J. Mech. Des. 2013, 135, 071002. [CrossRef]

4. Xu, S.W. Development Trend of Axial Piston Pump and Motor Hyd. J. Pneum. Seals 2003, 34, 10-15.

5. Shen, L.L.; Lu, P.F. Research on Friction Performance Study of Piston Pairs of Swash Plate Axial Piston Pump and Port Plate Pairs. J. Mach. Des. Manuf. 2014, 63, 167-173.

6. Ma, L.Y.; Tian, M.T.; Song, Y.B.; Li, R.W. The Force Analysis and Reliability Study on Friction Pairs for Ram-type Pump. J. Mod. Manuf. Technol. Equip. 2016, 115, 8-10.

7. Ma, J.M.; Huang, Y.H.; Guo, J.; Shi, Y.Y.; Song, Y.H. Review of Wear Analyses Research for Main Pairss in Hydraulic Axial Piston Pump. J. Chin. Hydraul. Pneum. 2017, 134, $42-47$.

8. Tan, G. Discussion on Ultra-high Pressure Hydraulic Technology in Construction Machinery. J. Sci. Technol. Inf. 2014, 38, 100-103.

9. Qiao, P.P. Discussed and Application of Super High Pressure Hydraulic Seal Method. J. Hydraul. Pneum. Seals 2014, 34, 31-35.

10. Yamaguchi, A.; Tanioka, Y. Motion of Pistons in Piston-Type Hydraulic Machines: 1st Report, Theoretical Analysis. J. Trans. Jpn. Soc. Mech. Eng. 1975, 41, 2399-2406. [CrossRef]

11. Tanaka, K.; Nakahara, T.; Kyogoku, K.; Momozono, S. Oil Whirl of Piston in Axial Piston Pump and Motor. Numerical Calculation under Mixed Lubrication. J. Trans. Jpn. Soc. Mech. Eng. 1998, 64, 653-661. [CrossRef]

12. Tanaka, K.; Nakahara, T.; Kyogoku, K. Experimental Verification of Oil Whirl of Piston in Axial Piston Pump and Motor. JSME Int. J. Ser. C Mech. Syst. Mach. Elem. Manuf. 2002, 44, 230-236. [CrossRef]

13. Tanaka, K.; Kyogoku, K.; Nakahara, T. Lubrication Characteristics on Sliding Surfaces between Piston and Cylinder in a Piston Pump and Motor. Effects of Running-In, Profile of Piston Top and Stiffness. J. Trans. Jpn. Soc. Mech. Eng. C 1998, 64, 3959-3967. [CrossRef] 
14. He, X.F.; Zhang, T.H.; Yang, S.D. An Experimental Research Method for Piston Pairss in Water Hydraulic Piston Pump. J. Hydraul. Pneum. Seals 2001, 2, 7-8.

15. Zhang, B. Study on Virtual Prototype and Pressure Characteristics of Oil Film for Axial Piston Pump. Ph.D. Thesis, Zhejiang University, Hangzhou, China, 2009.

16. Bergada, J.M.; Kumar, S.; Davies, D.L.; Watton, J. A complete analysis of axial piston pump leakage and output flow ripples. J. Appl. Math. Model. 2012, 36, 1731-1751. [CrossRef]

17. Ivantysynova, M.; Lasaar, R. An Investigation into Micro- and Macrogeometric Design of Piston/Cylinder Assembly of Swash Plate Machines. J. Int. Fluid Power 2004, 5, 23-36. [CrossRef]

18. Xu, B.; Zhang, J.H.; Yang, H.Y. Simulative analysis of piston-cylinder pairs of axial piston pump based on virtual prototype. J. Lanzhou Univ. Technol. 2010, 36, 31-37.

19. Lü, F.; Xu, B.; Zhang, J.H. Simulative Analysis of Piston Posture and Piston/Cylinder Interface Leakage of EHA Pumps by the Influence of Rotating Speed. J. Mech. Eng. 2018, 54, 123-130.

(C) 2020 by the authors. Licensee MDPI, Basel, Switzerland. This article is an open access article distributed under the terms and conditions of the Creative Commons Attribution (CC BY) license (http://creativecommons.org/licenses/by/4.0/). 